\title{
3D Conformal Re-Irradiation with Temozolamide for Recurrent Glioblastoma: A Prospective Cohort Study
}

\author{
Ghada Ezzat Eladawei ${ }^{\circ}$, Rasha Mohamed Abdellatif \\ Clinical Oncology and Nuclear Medicine Department, Mansoura University, Mansoura, Egypt \\ Email: ghadaeladawy@hotmail.com,rosha20075@yahoo.com
}

How to cite this paper: Eladawei, G.E. and Abdellatif, R.M. (2019) 3D Conformal Re-Irradiation with Temozolamide for Recurrent Glioblastoma: A Prospective Cohort Study. Journal of Cancer Therapy, 10, 619-631.

https://doi.org/10.4236/jct.2019.108051

Received: March 21, 2019

Accepted: July 29, 2019

Published: August 1, 2019

Copyright ( 2019 by author(s) and Scientific Research Publishing Inc. This work is licensed under the Creative Commons Attribution International License (CC BY 4.0).

http://creativecommons.org/licenses/by/4.0/

\begin{abstract}
Introduction and objectives: Salvage treatment of recurrent Glioblastoma (GBM) is one of the most challenging tasks in neuro-oncology. There is no standard treatment for recurrent GBM as options include resection, chemotherapy, and re-irradiation either separate or in combination. Role of concomitant temozolamide with re-irradiation in recurrent disease is still debatable. Therefore, this study evaluates efficacy of concurrent and adjuvant temozolamide with re-irradiation in management of recurrent GBM. Patients and methods: Twenty two patients with recurrent glioblastoma were eligible. Patients were treated with $3 \mathrm{D}$ conformal radiotherapy. The dose ranged from 30 to 40 Gy in 1.6 to $1.8 \mathrm{~Gy}$ per fraction for 5 days per week. Temozolamide was administrated at $50 \mathrm{mg} / \mathrm{m}^{2}$ daily dose during radiation therapy. Adjuvant Temozolomide $\left(200 \mathrm{mg} / \mathrm{m}^{2}\right)$ was given orally for five days every four weeks for 4 - 6 cycles for patients who did not receive temozolamide before, and 150 $\mathrm{mg} / \mathrm{m}^{2}$ for pretreated patients. Results: 22 patients received re-irradiation with median dose $38 \mathrm{~Gy}$ (range 33 - $40 \mathrm{~Gy}$ ), concurrent with temozolamide. The time interval between primary and re-irradiation ranged from 6 to 23 months with median 12 months. The re-irradiated volume, median was $101.95 \mathrm{~cm}^{3}$ (range $30-375 \mathrm{~cm}^{3}$ ). The median cumulative maximum dose to optic system and brain stem were $53.5 \mathrm{~Gy}$ (range 42 - $63 \mathrm{~Gy}$ ), and $60 \mathrm{~Gy}$ (range 54 - 73 Gy), respectively. Response rate was $72.7 \%$, one patient showed complete response (4.5\%), partial response and stable disease registered in $22.7 \%$ and $45.5 \%$, respectively. The median overall survival (OS) was 10 months (range 4 - 13 months), and median progression-free (PFS) survival was 7.5 months (range 2 - 11 months). The 6 and 12 months OS rate was $100 \%$ and $56.6 \%$ respectively, and the 6 months PFS rate was $93.3 \%$. No major acute toxicity was observed. About $70 \%$ of patients experienced grade 2 toxicity in the form of headache, nausea \& vomiting, skin erythema and alopecia. The late toxicity was minimal as GI \& II. Symptoms of radiation necro-
\end{abstract}


sis were not recorded in any patient. Conclusion: 3D conformal re-irradiation concomitant with temozolamide and adjuvant temozolamide appears effective treatment in recurrent glioblastoma. The treatment protocol is safe, feasible treatment with limited rate of toxicity and improve survival outcome.

\section{Keywords}

Recurrent Glioblastoma, Re-Irradiation, Temozolomide

\section{Introduction}

Glioblastoma (GBM) is the grade IV glioma [1] which is aggressive and carrys poor prognosis [2]. Treatment of GBM consisted of multimodality form and included surgical excision followed by radiotherapy and systemic treatment given concurrently and adjuvant. Although of this combined treatment, rate of recurrence is still high at median of 8 months [3].

There is no standard treatment for recurrent GBM as options include resection, chemotherapy, and re-irradiation either separate or in combination [4] [5].

Re-irradiation used cautiously as line of treatment of recurrent GBM because of risk of radionecrosis. It was first used in 1996 [6]. Improvement of imaging and radiation techniques as fractionated stereotactic radiotherapy (FSRT), protons, and intensity modulated radiotherapy (IMRT) allowed delivering conformal treatment with better efficacy and reduced toxicity and in turn helped use of re-irradiation in recurrent high-grade gliomas with acceptable improvement of survival [7] [8] [9] [10].

When re-irradiation was selected as a treatment of recurrent GBM it is found that results were better with selection of patients with specific risk factors as age, performance status, size of re-irradiated volume, interval passes after initial irradiation, and resection of recurrent lesion [11].

Use of systemic therapy with re-irradiation in recurrent high grades gliomas was controversial. Some studies failed to get benefit of giving chemotherapy with re-irradiation [10] [12]. Others proved improved outcome with addition of bevacizumab to re-irradiation with good tolerability [13] [14].

Temozolamide being proved to improve outcome in GBM when used as concurrent and adjuvant with radiotherapy in primary tumors, enforced researchers to study it in recurrent, even in patients who previously used temozolamide in initial treatment, and resulted in improvement in objective response and stability of disease [15] [16].

In this prospective study we try to evaluate the efficacy of concurrent and adjuvant temozolamide with re-irradiation in recurrent GBM considering response as primary endpoint and survival together with toxicity as secondary endpoint.

\section{Patients \& Methods}

After approval by Institutional Review Board of Mansoura faculty of Medicine 
(IRB-MFM), this is prospective phase II trial was conducted in Clinical Oncology \& nuclear Medicine department, Mansoura University Hospital between January 2015 and January 2018.

\section{Study objectives}

The primary objective of this study was evaluation of efficacy of concurrent and adjuvant temozolamide with re-irradiation in management of recurrent GBM.

The secondary objectives were overall survival, progression-free survival and treatment related toxicity.

\section{Inclusion criteria}

Histologically proven Glioblastoma with evidence of tumor recurrence, Eastern Cooperative Oncology Group (ECOG) performance status $\leq 2$, aged above 18 and below 70 years and had adequate liver, kidney and hematological functions. All patients must receive radiotherapy as apart from their initial treatment. An interval of at 6 months must pass since completion of previous radiation course. Consent was taken from all patients before inclusion into the study.

\section{Definition of recurrence}

Recurrence defined as appearance of new enhanced lesion on MRI or increase in the size of the initial lesion according to the MacDonald's criteria [17]. If the diagnosis of true progression (in contrast to pseudoprogression) was uncertain, MRI repeated after three months. Symptomatic patients suspected to have pseudoprogression were kept on steroids and MRI was repeated after one month.

\section{Treatment protocol}

Patients were treated with $3 \mathrm{D}$ conformal radiotherapy. The dose ranged from 30 to 40 Gy in 1.6 to 1.8 Gy per fraction for 5 days per week. Temozolamide was administrated at $50 \mathrm{mg} / \mathrm{m}^{2}$ daily dose during radiation therapy.

Adjuvant Temozolomide $\left(200 \mathrm{mg} / \mathrm{m}^{2}\right)$ was given orally for five days every four weeks for 4 - 6 cycles for patients who did not receive temozolamide before, $150 \mathrm{mg} / \mathrm{m}^{2}$ for pretreated patients.

\section{Re-irradiation 3D conformal radiotherapy technique}

Patients were planned via $3 \mathrm{D}$ conformal radiotherapy. Patients planned in supine position and immobilized with thermoplastic devices. CT planning was done every $1-3 \mathrm{~mm}$ transverse sections from vertex till C4 vertebra.

\section{Target volume definitions}

Target volume was defined by CT treatment planning in corporation with MRI data. Gross tumor volume (GTV) was delineated as contrast enhancing tumor in CT images with T1-images on MRI. Clinical target volume (CTV) was defined by $\mathrm{T} 2$ weighed and FLAIR images of MRI. The planning target volume (PTV) was defined by adding $1 \mathrm{~cm}$ to GTV to include surrounding oedema. PTV was reduced in areas near organ at risks. The organs at risk included optic chiasm, optic nerves, eyes and brain stem. The organ at risk and previously high dose irradiated volumes were delineated and during planning care was undertaken to decrease dose to these structures. The cumulative dose to optic chiasm, 
optic nerves and brain stem were reported from a composite plan of re-irradiation treatment and primary radiation treatment plan. The guide of treatment planning is towards safety. The target dose is reduced if needed.

\section{Follow-up \& toxicity evaluation}

Baseline neurological examination and MRI brain were performed before treatment. During treatment protocol, patients were closely followed twice weekly for treatment-related toxicities or any complaints. CBC, liver function and serum creatinine were required before subsequent chemotherapy cycles.

During radiotherapy, patients were kept on dexamethasone $8 \mathrm{mg}$ orally, every 12 hours, with proton pump inhibitor $20 \mathrm{mg}$ orally, twice daily, and antiepileptic prophylaxis.

After treatment completion, patients were evaluated for both subjective and objective response (Table 1 ) by history, physical examination, laboratory investigation and radiological studies. Patients were followed up regularly as routine follow up visits every month for the first six months then every 3 months thereafter.

MRI brain was done after treatment protocol, and every 2 months in first 6 months, then every 3 months thereafter for follow up.

\section{Toxicity}

Toxicity was graded as per Common Terminology Criteria for adverse events (CTCAE) version 4.

\section{Statistical analysis}

Descriptive statistics will be provided to summarize the patient characteristics and toxicities. Overall survival (OS) was calculated from the date of re-irradiation to date of death or last follow up. Progression-free survival (PFS) was calculated from time of re-irradiation until further tumor progression or death, whichever occurred earlier. Qualitative data were presented as number and percent. Non-parametric data was presented as min - max and median. Survival calculated using Kaplan-Meier method. Univariate survival analysis was performed with long rank test. Multivariate Cox regression analysis was performed using OS as outcomes with a significance level of $\mathrm{P}<0.05$. Statistical Package for Social Sciences (SPSS) version 20 is used for statistical analysis.

\section{Results}

This is a prospective phase II study included 22 patients attended to Clinical

Table 1. Response definitions.

\begin{tabular}{cc}
\hline Response & Definitions \\
\hline Complete response (CR) & Disappearance of all contrast-enhancing tumors. \\
Partial response (PR) & $50 \%$ or more reduction in the size of measurable disease. \\
Disease progression (DP) & $25 \%$ or more increase in the size of measurable disease. \\
Stable disease (SD) & All other situations.
\end{tabular}


Oncology \& Nuclear Medicine department of Mansoura University Hospital in the period between January 2015 and January 2018.

Patients' characteristics and treatment details:

The patients' characteristics showed in Table 2. The median age of all patients is 51.5 years with range 20 - 65 years, $63.6 \%$ of patients were in age group $>45$ years. Sixteen patients were male (72.7\%), with male to female ratio 2.6:1. Most of patients (68.2\%) presented with ECOG1 performance status. Seventeen patients (77.3\%) were operated with subtotal excision of primary tumor. Fourteen patients $(63.6 \%)$ received temozolamide as concurrent treatment with primary irradiation. The median initial irradiation dose was $60 \mathrm{~Gy}$.

All patients received re-irradiation with median dose 38 Gy (range 33 - 40 Gy), concurrent with temozolamide. The interval time between primary and re-irradiation ranged from 6 to 23 months with median 12 months. As regard the re-irradiated volume, the median volume was $101.95 \mathrm{~cm}^{3}$ (range $30-375$ $\mathrm{cm}^{3}$ ). The median cumulative maximum dose to optic system and brain stem were $53.5 \mathrm{~Gy}$ (range 42 - $63 \mathrm{~Gy}$ ), and $60 \mathrm{~Gy}$ (range 54 - 73 Gy), respectively (Table 3).

Table 2. Patients' characteristics.

\begin{tabular}{|c|c|c|}
\hline Characteristics & Number $(n=22)$ & Percentage (\%) \\
\hline \multicolumn{3}{|l|}{ Age } \\
\hline \multicolumn{3}{|l|}{ median 51.5 (range 20 - 65) } \\
\hline$\leq 45$ & 8 & $36.4 \%$ \\
\hline$>45$ & 14 & $63.6 \%$ \\
\hline \multicolumn{3}{|l|}{ Sex } \\
\hline Male & 16 & $72.7 \%$ \\
\hline Female & 6 & $27.3 \%$ \\
\hline \multicolumn{3}{|l|}{ ECOG performance status } \\
\hline 1 & 15 & $68.2 \%$ \\
\hline 2 & 7 & $31.8 \%$ \\
\hline \multicolumn{3}{|l|}{ Site of primary tumors } \\
\hline frontal & 7 & $31.8 \%$ \\
\hline temporal & 14 & $63.6 \%$ \\
\hline parietal & 15 & $68.2 \%$ \\
\hline occipital & 2 & $9.1 \%$ \\
\hline ventricular & 1 & $4.5 \%$ \\
\hline \multicolumn{3}{|l|}{ Primary surgery } \\
\hline Biopsy & 3 & $13.6 \%$ \\
\hline Subtotal excision & 17 & $77.3 \%$ \\
\hline Maximal safe resection & 2 & $9.1 \%$ \\
\hline \multicolumn{3}{|l|}{ Primary systemic treatment } \\
\hline Temozolamide & 14 & $63.6 \%$ \\
\hline PCV protocol & 8 & $36.4 \%$ \\
\hline
\end{tabular}

(ECOG) Eastern Cooperative Oncology Group, PCV protocol (procarbazine, lomustine, vincristine). 
Table 3. Treatment information.

\begin{tabular}{cccc}
\hline Treatment information & Median & Minimum & Maximum \\
\hline Primary radiation dose & 60 & 55 & 60 \\
Re-irradiation dose (Gy) & 38 & 33 & 40 \\
Time interval to re-irradiation (month) & 12 & 6 & 23 \\
Re-irradiation volume ( $\left.\mathrm{cm}^{3}\right)$ & 101.95 & 30 & 374 \\
Cumulative maximum brain stem dose (Gy) & 60 & 54 & 73 \\
Cumulative maximum optic apparatus dose (Gy) & 53.5 & 42 & 63 \\
\hline
\end{tabular}

\section{Response data}

Response rate was $72.7 \%$, one patient showed complete response (4.5\%), partial response and stable disease registered in $22.7 \%$ and $45.5 \%$, respectively (Table 4).

\section{Survival Outcomes:}

The median OS was 10 months (range 4 - 13 months), and median PFS was 7.5 months (range 2 - 11 months). The 6 and 12 months OS rate was $100 \%$ and 56.6\% respectively (Figure 1), and the 6 months PFS rate was 93.3\% (Figure 2).

Cox regression analysis of prognostic factors affecting survival was performed. On univariate analysis, better survival was observed in younger age, male gender, previously excised in primary treatment with (median OS 11, 11, 10.5 months respectively) but not statistically significant $(\mathrm{P}=0.105,0.101,0.626$, respectively). However Performance status was the only factor associated with statistically significant difference (median 11 months, $\mathrm{P}=0.036$ ). OS was equivalent in time interval $\leq 12$ and $>12$ months and in treatment volume $\leq 100$ and $>100 \mathrm{~cm}^{3}$ (median 10, 10 months with $\mathrm{P}=0.407$, and 0.691, respectively) (Table 5). Multivariate analysis of prognostic factors affect OS, (age, sex, previously excised primary tumor, performance status, time interval, and treatment volume) no factor associated with any significance even Performance status $(P=0.123$, $0.114,0.789,0.064,0.543,0.712$, respectively).

\section{Treatment toxicity}

No major acute toxicity observed (no grade III, nor grade IV), according to CTCAE version 4 . About $70 \%$ of patients experienced grade II toxicity in the form of headache, nausea \& vomiting, skin erythema and alopecia. The late toxicity was minimal as grade I \& II. Symptoms of radiation necrosis were not recorded in any patient (Table 6).

The toxicity related to temozolamide was in the form of grade I nausea/vomiting, anaemia, thrompocytopenia, but not associated with delayed cycle or reduction of dose and not associated with increases radiation toxicity.

\section{Discussion}

Salvage treatment of recurrent Glioblastoma is one of the most challenging tasks in neuro-oncology [18]. Re-irradiation has been widely accepted as useful therapeutic option in treatment of recurrent Glioblastoma [19] [20]. Stereotactic 


\section{Survival Function}

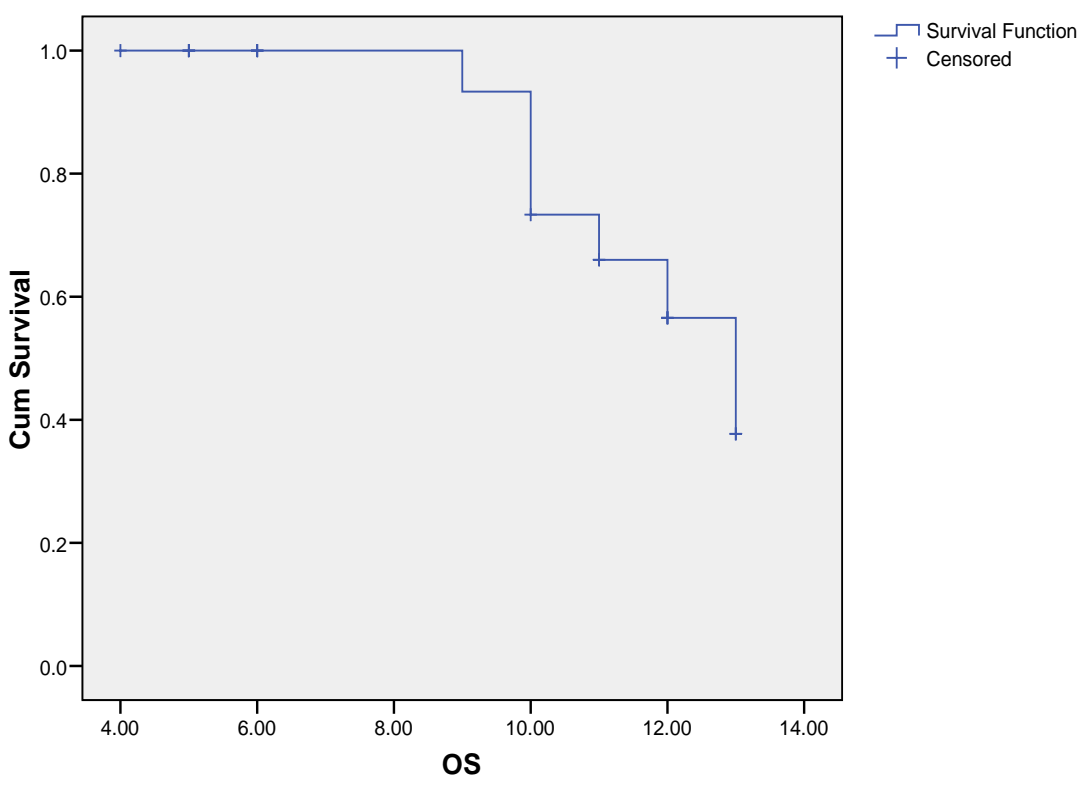

Figure 1. Kaplan-Meier curve for overall survival.

\section{Survival Function}

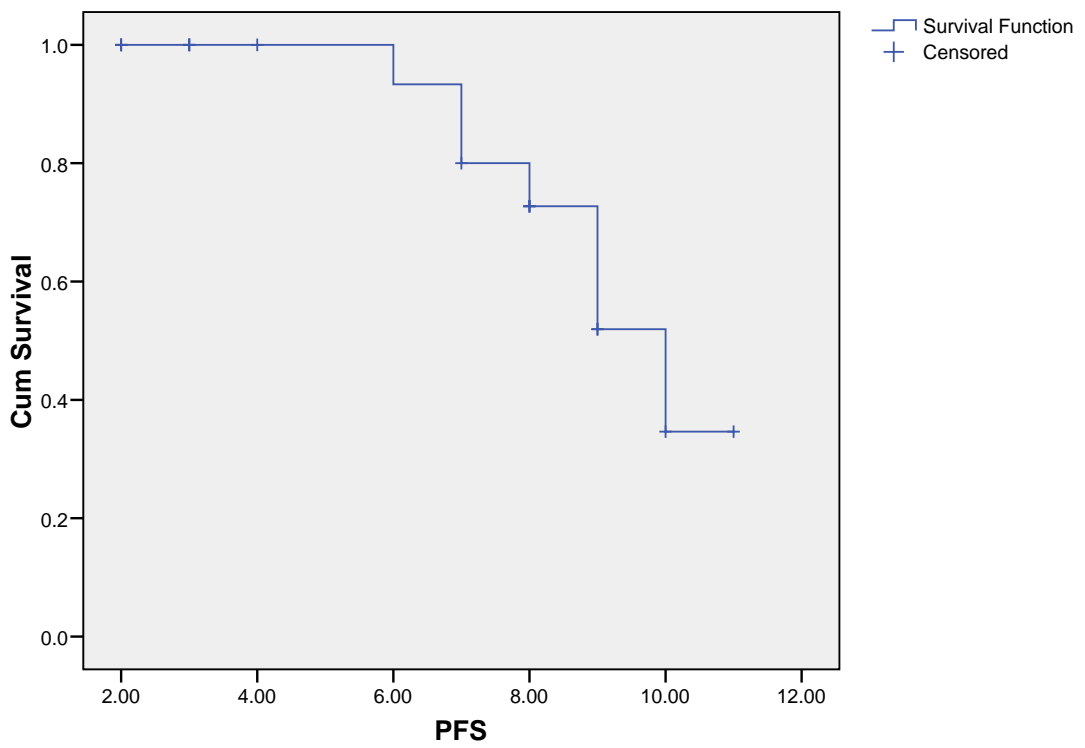

Figure 2. Kaplan-Meier curve for progression free survival.

Table 4. Response of tumor to treatment protocol.

\begin{tabular}{ccc}
\hline Response & Number of patients & Percentage (\%) \\
\hline Complete response (CR) & 1 & $4.5 \%$ \\
Partial response (PR) & 5 & $22.7 \%$ \\
Stationary disease (SD) & 10 & $45.5 \%$ \\
Progressive disease (PD) & 6 & $27.3 \%$ \\
\hline
\end{tabular}


Table 5. Univariate analysis of factors predicting overall survival (OS).

\begin{tabular}{|c|c|c|}
\hline Factors & Median OS (months), range & $P$ value \\
\hline \multicolumn{3}{|l|}{ Age } \\
\hline$\leq 45$ & $11(10-13)$ & 0.105 \\
\hline$>45$ & $7.5(4-13)$ & \\
\hline \multicolumn{3}{|l|}{ Sex } \\
\hline Male & $11(4-13)$ & 0.101 \\
\hline female & $8(5-11)$ & \\
\hline \multicolumn{3}{|c|}{ ECOG performance status } \\
\hline 1 & $11(6-13)$ & 0.036 \\
\hline 2 & $5(4-12)$ & \\
\hline \multicolumn{3}{|c|}{ Primary surgical treatment } \\
\hline Biopsy & $6(4-12)$ & \\
\hline Subtotal excision & $10(5-13)$ & 0.020 \\
\hline Total excision & $10.5(10-11)$ & \\
\hline \multicolumn{3}{|c|}{ Primary systemic treatment } \\
\hline Temozolamide & $11.5(6-13)$ & 0.138 \\
\hline CVP & $10(4-13)$ & \\
\hline \multicolumn{3}{|l|}{ Time interval } \\
\hline$\leq 12$ month & $10(4-13)$ & 0.407 \\
\hline$>12$ month & $10(6-13)$ & \\
\hline \multicolumn{3}{|l|}{ Re-irradiation volume } \\
\hline$\leq 100 \mathrm{~cm}^{3}$ & $10(4-13)$ & 0.691 \\
\hline$>100 \mathrm{~cm}^{3}$ & $10(5-13)$ & \\
\hline
\end{tabular}

Table 6. Acute and late toxicity.

\begin{tabular}{ccc}
\hline Toxicity & Number & $\%$ \\
\hline Acute toxicity & 4 & $18.2 \%$ \\
Grade 0 & 3 & $13.6 \%$ \\
Grade 1 & 15 & $68.2 \%$ \\
Grade 2 & 0 & $0 \%$ \\
Grade 3 & & \\
Late toxicity & 8 & $36.4 \%$ \\
Grade 0 & 5 & $22.7 \%$ \\
Grade 1 & 9 & $40.9 \%$ \\
Grade 2 & 0 & $0 \%$ \\
Grade 3 & 5 & \\
\hline
\end{tabular}


radiosurgery and hypofractionated stereotactic radiation are limited to recurrence with small volumes [21] [22]. Conventional fractionated radiotherapy is well tolerated for larger recurrent volumes [9] [23].

Temozolamide is an effective agent as first line treatment in the vast majority of patients with recurrent high-grade glioma [24]. Role of concomitant temozolamide with re-irradiation is well established in primary treatment of high-grade glioma [3]. On the other hand its value in recurrent disease is still debatable in those patients who received prior temozolamide in their initial treatment and potential development of resistance [25].

Researchers commonly used temozolamide alone for median eight cycles or combined temozolamide with other agents including radiation for median four to six cycles aiming to achieve survival benefit and good objective response [26]. Furthermore, Van den Bent et al. observed that administration of temozolamide more than six cycles may be associated with more toxicity [27].

The current study included 22 patients with recurrent Glioblastoma. They treated with concurrent temozolamide with re-irradiation (median dose $38 \mathrm{~Gy}$ ) followed by 4 - 6 cycles of temozolamide. The current protocol achieved response rate 72.7\% (CR 4.5\%, PR 22.7\%, SD 45.5\%). Similarly, Kataria et al. [28] retrospectively analyzed 25 patients with recurrent glioblastoma. Patients were treated with re-irradiation concomitant temozolamide and adjuvant temozolamide. Re-irradiation methods included stereotactic radiosurgery for 2 patients, hypofractionated stereotatic radiation therapy ( $15-40$ Gy in $3-5$ fraction) for 14 patients and conventional fractionated radiotherapy (45 - 54 Gy in $25-27$ fraction). Tumor response rate was observed in $84 \%$ of patients.

Greenspoon et al. [29] prospectively assessed 31 patients with recurrent glioblastoma. All patients were treated with fractionated stereotactic radiosurgery 25 to $35 \mathrm{~Gy}$ in five fraction concomitant with temozolamide $75 \mathrm{mg} / \mathrm{m}^{2}$. They observed $60 \%$ progression-free survival in 6 months. Another prospective study using 4 - 6 cycles temozolamide followed by re-irradiation with 3D conformal radiotherapy (30 - 40 Gy) in recurrent high-grade glioma (grade III and IV). Overall response rate was $20.6 \%$ and stable disease was $45 \%$ [30].

The current treatment protocol was well tolerated, with mild side effects. No grade III or IV toxicities were observed in the patients following re-irradiation during follow up period. Temozolamide related toxicity was mild and relieved by supportive treatment. Similar observation was reported in a study conducted by Kataria et al. [28]. Another retrospective study assessed outcome of 118 patients with recurrent or progressive high grade glioma. Patients were treated with conventional radiotherapy of median re-irradiation dose $41.4 \mathrm{~Gy} .56 \%$ of patients received temozolamide with re-irradiation. The authors reported that there is no symptomatic persistant brain stem or optic chiasma injury. Grade $\geq 3$ late toxicity and radiation necrosis were minimal (less than 5\%) [31].

Radionecrosis was not reported in the current study. Flickinger et al. [32] assessed the probability of necrosis at different stereotactic radiosurgery doses in different versions of integrated logistic formula. Dose volume histograms gener- 
ate only 3\% risk of necrosis. Normalized total dose of conventional re-irradiation was lower than those used in either stereotactic radiosurgery or fractionated stereotactic radiotherapy [33]. Radionecrosis happened at normalized total doses more than $100 \mathrm{~Gy}$. There was no association between risk of radionecrosis and interval time between treatment courses. Due to limiting normal tissue irradiation, re-irradiation using stereotactic and conformal techniques is safe and associated with limited risk of radionecrosis [34].

The current study demonstrated median overall survival of 10 months and The 6 and 12 months OS rate was $100 \%$ and 56.6\% respectively. Median progression-free survival was 7.5 months and 6 months PFS rate was $93.3 \%$., Which relatively better than those reported by Minniti et al. [35].

Minniti et al. [35] reported on 36 patients with recurrent glioblastoma. Patient received fractionated stereotactic radiotherapy (37.5 Gy in 15 fractions over 3 weeks) with concomitant daily temozolamide $75 \mathrm{mg} / \mathrm{m}^{2}$. Median overall survival was 9.7 months the 6- and 12-month survival rates were $84 \%$ and $33 \%$, and 5 months of median PFS 6- and 12-month PFS rates were $42 \%$ and $8 \%$.

Conti et al. [36] treated 23 patients with Cyberknife stereotactic radiosurgery (median dose $20 \mathrm{~Gy}$ in two fractions). Twelve patients received concurrent 0temozolamide. The authors reported 12 months median overall survival for combined modality versus 7 months for stereotactic radiosurgery alone. 6 month progression-free survival was $66.7 \%$ for combined treatment versus $18 \%$ for radiosurgery alone. Similarly, Grosu et al. observed that fractionated stereotactic radiotherapy in combination with temozolamide significantly improve survival compared with fractionated stereotactic radiotherapy alone (11 months versus 6 months respectively) [37].

\section{Conclusion}

Despite that this study had limited number of cases, 3D conformal re-irradiation concomitant with temozolamide and adjuvant temozolamide appears effective treatment in recurrent glioblastoma. The treatment protocol is safe, feasible treatment with limited rate of toxicity and improve survival outcome.

\section{Conflicts of Interest}

The authors declare no conflicts of interest regarding the publication of this paper.

\section{References}

[1] Louis, D.N., Ohgaki, H., Wiestler, O.D., Cavenee, W.K., Burger, P.C., Jouvet, A., Scheithauer, B.W. and Kleihues, P. (2007) The 2007 WHO Classification of Tumours of the Central Nervous System. Acta Neuropathologica, 114, 97-109. https://doi.org/10.1007/s00401-007-0243-4

[2] Brandes, A.A. (2003) State-of-the-Art Treatment of High-Grade Brain Tumors. Seminars in Oncology, 30, 4-9. https://doi.org/10.1053/j.seminoncol.2003.11.028

[3] Stupp, R., Mason, W.P., van den Bent, M.J., Weller, M., Fisher, B., Taphoorn, M.J., 
Belanger, K., Brandes, A.A., Marosi, C., Bogdahn, U., Curschmann, J., Janzer, R.C., Ludwin, S.K., Gorlia, T., Allgeier, A., Lacombe, D., Cairncross, J.G., Eisenhauer, E. and Mirimanoff, R.O. (2005) Radiotherapy Plus Concomitant and Adjuvant Temozolomide for Glioblastoma. The New England Journal of Medicine, 352, 987-996. https://doi.org/10.1056/NEJMoa043330

[4] Birk, H.S., Han, S.J. and Butowski, N.A. (2017) Treatment Options for Recurrent High-Grade Gliomas. CNS Oncology, 6, 61-70.

https://doi.org/10.2217/cns-2016-0013

[5] Howard, S.P., Krauze, A., Chan, M.D., Tsien, C. and Tome, W.A. (2017) The Evolving Role for Re-Irradiation in the Management of Recurrent Grade 4 Glioma. Journal of Neuro-Oncology, 134, 523-530.

https://doi.org/10.1007/s11060-017-2392-1

[6] Bauman, G.S., Sneed, P.K., Wara, W.M., Stalpers, L.J., Chang, S.M., McDermott, M.W., Gutin, P.H. and Larson, D.A. (1996) Reirradiation of Primary CNS Tumors. International Journal of Radiation Oncology, Biology, Physics, 36, 433-441. https://doi.org/10.1016/S0360-3016(96)00315-X

[7] Combs, S.E., Gutwein, S., Thilmann, C.h., Huber, P., Debus, J. and Schulz-Ertner, D. (2005) Stereotactically Guided Fractionated Re-Irradiation in Recurrent Glioblastoma Multiforme. Journal of Neuro-Oncology, 74, 167-171.

https://doi.org/10.1007/s11060-004-2463-y

[8] Combs, S.E., Thilmann, C., Edler, L., Debus, J. and Schulz-Ertner, D. (2005) Efficacy of Fractionated Stereotactic Reirradiation in Recurrent Gliomas: Long-Term Results in 172 Patients Treated in a Single Institution. Journal of Clinical Oncology, 23, 8863-8869. https://doi.org/10.1200/JCO.2005.03.4157

[9] Flieger, M., Ganswindt, U., Schwarz, S.B., Kreth, F.W., Tonn, J.C., la Fougère, C., Ertl, L., Linn, J., Herrlinger, U., Belka, C. and Niyazi, M. (2014) Re-Irradiation and Bevacizumab in Recurrent High-Grade Glioma: An Effective Treatment Option. Journal of Neuro-Oncology, 117, 337-345. https://doi.org/10.1007/s11060-014-1394-5

[10] Fogh, S.E., Andrews, D.W., Glass, J., Champ, C., Evans, J.J., Hyslop, T., Pequignot, E., Downes, B., Comber, E., Maltenfort, M., Dicker, A.P. and Werner-Wasik, M. (2010) Hypofractionated Stereotactic Radiation Therapy: An Effective Therapy for Recurrent High-Grade Gliomas. Journal of Clinical Oncology, 28, 3048-3053.

https://doi.org/10.1200/JCO.2009.25.6941

[11] Scholtyssek, F., Zwiener, I., Schlamann, A., Seidel, C., Meixensberger, J., Bauer, M., Hoffmann, K.T., Combs, S.E., von Bueren, A.O., Kortmann, R.D., Müller K Seidel, C., Meixensberger, J., Bauer, M., Hoffmann, K.T., Combs, S.E., von Bueren, A.O., Kortmann, R.D. and Müller, K. (2013) Reirradiation in Progressive High-Grade Gliomas: Outcome, Role of Concurrent Chemotherapy, Prognostic Factors and Validation of a New Prognostic Score with an Independent Patient Cohort. Radiation Oncology, 8, 161. https://doi.org/10.1186/1748-717X-8-161

[12] Fokas, E., Wacker, U., Gross, M.W., Henzel, M., Encheva, E. and Engenhart-Cabillic, R. (2009) Hypofractionated Stereotactic Reirradiation of Recurrent Glioblastomas: A Beneficial Treatment Option after High-Dose Radiotherapy? Strahlentherapie und Onkologie, 185, 235-240. https://doi.org/10.1007/s00066-009-1753-x

[13] Gutin, P.H., Iwamoto, F.M., Beal, K., Mohile, N.A., Karimi, S., Hou, B.L., Lymberis, S., Yamada, Y., Chang, J. and Abrey, L.E. (2009) Safety and Efficacy of Bevacizumab with Hypofractionated Stereotactic Irradiation for Recurrent Malignant Gliomas. International Journal of Radiation Oncology, Biology, Physics, 75, 156-163. 
https://doi.org/10.1016/j.ijrobp.2008.10.043

[14] Cuneo, K., Vredenburgh, J., Sampson, J.H., Reardon, D.A., Desjardins, A., Peters, K.B., Friedman, H.S., Willett, C.G. and Kirkpatrick, J.P. (2012) Safety and Efficacy of Stereotactic Radiosurgery and Adjuvant Bevacizumab in Patients with Recurrent Malignant Gliomas. International Journal of Radiation Oncology, Biology, Physics, 82, 2018-2024.

[15] Franceschi, E., Omuro, A.M., Lassman, A.B., Demopoulos, A., Nolan, C. and Abrey, L.E. (2005) Salvage Temozolomide for Prior Temozolomide Responders. Cancer, 104, 2473-2476. https://doi.org/10.1002/cncr.21564

[16] Norden, A.D., Lesser, G.J., Drappatz, J., Ligon, K.L., Hammond, S.N., Lee, E.Q., Reardon, D.R., Fadul, C.E., Plotkin, S.R., Batchelor, T.T., Zhu, J.J., Beroukhim, R., Muzikansky, A., Doherty, L., Lafrankie, D., Smith, K., Tafoya, V., Lis, R., Stack, E.C., Rosenfeld, M.R. and Wen, P.Y. (2013) Phase 2 Study of Dose-Intense Temozolomide in Recurrent Glioblastoma. Neuro-Oncology, 15, 930-935.

https://doi.org/10.1093/neuonc/not040

[17] Macdonald, D.R., Cascino, T.L., Schold Jr., S.C. and Cairncross, J.G. (1990) Response Criteria for Phase II Studies of Supratentorial Malignant Glioma. Journal of Clinical Oncology, 8, 1277-1280. https://doi.org/10.1200/JCO.1990.8.7.1277

[18] Niyazi, M., Jansen, N., Ganswindt, U., Schwarz, S.B., Geisler, J., Schnell, O., Büsing, K., Eigenbrod, S., la Fougère, C. and Belka, C. (2012) Re-Irradiation in Recurrent Malignant Glioma: Prognostic Value of $\left[{ }^{18} \mathrm{~F}\right]$ FET-PET. Journal of Neuro-Oncology, 110, 389-395. https://doi.org/10.1007/s11060-012-0980-7

[19] Amichetti, M. and Amelio, D. (2011) A Review of the Role of Re-Irradiation in Recurrent High-Grade Glioma (HGG). Cancers, 3, 4061-4089. https://doi.org/10.3390/cancers3044061

[20] Nieder, C., Andratschke, N.H. and Grosu, A.L. (2016) Re-Irradiation for Recurrent Primary Brain Tumors. Anticancer Research, 36, 4985-4995. https://doi.org/10.21873/anticanres.11067

[21] Combs, S.E., Widmer, V., Thilmann, C., Hof, H., Debus, J. and Schulz-Ertner, D. (2005) Stereotactic Radiosurgery (SRS). Cancer, 104, 2168-2173. https://doi.org/10.1002/cncr.21429

[22] Shepherd, S.F., Laing, R.W., Cosgrove, V.P., Warrington, A.P., Hines, F., Ashley, S.E. and Brada, M. (1997) Hypofractionated Stereotactic Radiotherapy in the Management of Recurrent Glioma. International Journal of Radiation Oncology, Biology, Physics, 37, 393-398. https://doi.org/10.1016/S0360-3016(96)00455-5

[23] Niyazi, M., Ganswindt, U., Schwarz, S.B., Kreth, F.W., Tonn, J.C., Geisler, J., la Fougère, C., Ertl, L., Linn, J., Siefert, A. and Belka, C. (2012) Irradiation and Bevacizumab in High-Grade Glioma Retreatment Settings. International Journal of Radiation Oncology, Biology, Physics, 82, 67-76. https://doi.org/10.1016/j.ijrobp.2010.09.002

[24] Weller, M., Cloughesy, T., Perry, J.R. and Wick, W. (2013) Standards of Care for Treatment of Recurrent Glioblastoma-Are We There Yet? Neuro-Oncology, 15, 4-27. https://doi.org/10.1093/neuonc/nos273

[25] Messaoudi, K., Clavreul, A. and Lagarce, F. (2015) Toward an Effective Strategy in Glioblastoma Treatment. Part I: Resistance Mechanisms and Strategies to Overcome Resistance of Glioblastoma to Temozolomide. Drug Discovery Today, 20, 899-905. https://doi.org/10.1016/j.drudis.2015.02.011

[26] Prados, M.D., Yung, W.K., Fine, H.A., Greenberg, H.S., Junck, L., Chang, S.M., Nicholas, M.K., Robins, H.I., Fink, K.L., Mehta, M.P., Jaeckle, K.A., Kuhn, J., Hess, 
K.R. and Schold Jr., S.C. (2004) Phase 2 Study of BCNU and Temozolomide for Recurrent Glioblastoma Multiforme: North American Brain Tumor Consortium study. Neuro-Oncology, 6, 33-37. https://doi.org/10.1215/S1152851703000309

[27] van den Bent, M.J., Keime-Guibert, F., Brandes, A.A., Taphoorn, M.J., Kros, J.M., Eskens, F.A. and Carpentier, A.F. (2001) Temozolomide Chemotherapy in Recurrent Oligodendroglioma. Neurology, 57, 340-342.

https://doi.org/10.1212/WNL.57.2.340

[28] Kataria, T., Gupta, D., Gupta, R., Narang, K. and Banerjee, S. (2017) Recurrent Glioblastoma: A Single-Institution Experience with Reirradiation and Temozolomide. Journal of Radiation Oncology, 6, 133-141.

https://doi.org/10.1007/s13566-017-0307-6

[29] Greenspoon, J.N., Sharieff, W., Hirte, H., Overholt, A., Devillers, R., Gunnarsson, T. and Whitton, A. (2014) Fractionated Stereotactic Radiosurgery with Concurrent Temozolomide Chemotherapy for Locally Recurrent Glioblastoma Multiforme: A Prospective Cohort Study. OncoTargets and Therapy, 7, 485-490. https://doi.org/10.2147/OTT.S60358

[30] Osman, M.A.M. (2014) Phase II Trial of Temozolomide and Reirradiation Using Conformal 3D-Radiotherapy in Recurrent Brain Gliomas. Annals of Translational Medicine, 2, 44.

[31] Shen, C.J., Kummerlowe, M.N., Redmond, K.J., Martinez-Gutierrez, J.C., Usama, S.M., Holdhoff, M., Grossman, S.A., Laterra, J.J., Strowd, R.E. and Kleinberg, L.R. (2018) Re-Irradiation for Malignant Glioma: Toward Patient Selection and Defining Treatment Parameters for Salvage. Advances in Radiation Oncology, 3, 582-590. https://doi.org/10.1016/j.adro.2018.06.005

[32] Flickinger, J., Schell, M. and Larson, D. (1990) Estimation of Complications for Linear Accelerator Radiosurgery with the Integrated Logistic Formula. International Journal of Radiation Oncology, Biology, Physics, 19, 143-148. https://doi.org/10.1016/0360-3016(90)90146-B

[33] Mayer, R. and Sminia, P. (2008) Reirradiation Tolerance of the Human Brain. International Journal of Radiation Oncology, Biology, Physics, 70, 1350-1360. https://doi.org/10.1016/j.ijrobp.2007.08.015

[34] Sminia, P. and Mayer, R. (2012) External Beam Radiotherapy of Recurrent Glioma: Radiation Tolerance of the Human Brain. Cancers, 4, 379-399. https://doi.org/10.3390/cancers4020379

[35] Minniti, G., Armosini, V., Salvati, M., Lanzetta, G., Caporello, P., Mei, M., Osti, M.F. and Maurizi, R.E. (2011) Fractionated Stereotactic Reirradiation and Concurrent Temozolomide in Patients with Recurrent Glioblastoma. Journal of Neuro-Oncology, 103, 683-691. https://doi.org/10.1007/s11060-010-0446-8

[36] Conti, A., Pontoriero, A., Arpa, D., Siragusa, C., Tomasello, C., Romanelli, P., Cardali, S., Granata, F., De Renzis, C. and Tomasello, F. (2012) Efficacy and Toxicity of CyberKnife Re-Irradiation and "Dose Dense" Temozolomide for Recurrent Gliomas. Acta Neurochirurgica, 154, 203-209. https://doi.org/10.1007/s00701-011-1184-1

[37] Grosu, A.L., Weber, W.A., Franz, M., Stärk, S., Piert, M., Thamm, R., Gumprecht, H., Schwaiger, M., Molls, M. and Nieder, C. (2005) Reirradiation of Recurrent High-Grade Gliomas Using Amino Acid PET (SPECT)/CT/MRI Image Fusion to Determine Gross Tumor Volume for Stereotactic Fractionated Radiotherapy. International Journal of Radiation Oncology, Biology, Physics, 63, 511-519. https://doi.org/10.1016/j.ijrobp.2005.01.056 